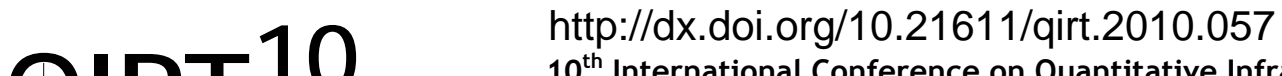 \\ $10^{\text {th }}$ International Conference on Quantitative InfraRed Thermography \\ July 27-30, 2010, Québec (Canada)
}

\section{Multimodal Defect Quantification}

\author{
* Fraunhofer Development Center for X-ray Technology EZRT, Dr.-Mack-Str. 8190762 Fuerth,
} stefanie.huebner@iis.fraunhofer.de,

\begin{abstract}
In the last few years thermographic testing methods gained much importance in the testing of composite materials such as carbon reinforced polymers (CFRP). In many cases the detectabiliy of defects like voids, cracks or debonding has been proofed. Up to now the quantitative analysis of these defects is limited. First approaches are done here by profilometric calculation for the depth, but lateral quantification is still difficult due to thermal diffusion. Herein we present a method, which allows to measure the dimensions and the depth of defects by combining thermography and X-ray testing.
\end{abstract}

\section{Introduction}

In modern industrial production components are to be built using less material and having less weight in order to reduce costs while at the same time keeping up and even enhancing the reliability during the part's lifetime. Carbon fiber reinforced polymers (CFRP) are groundbreaking materials in aerospace applications with a high ratio of strength to weight, but they also impose a new challenge for inspection. As non-destructive testing techniques are mostly used in safety relevant structures, the detection of subsurface boundaries and cracks is essential. Due to the increasing complexity of the CFRP parts, a single testing method alone is sometimes not sufficient. As the various inspection methods are sensitive to different defects and they provide different geometric information, combining physically different methods is expected to improve the reliability and interpretability of inspection.

Therefore we perform an image fusion of depth resolved thermography with radioscopy, in which the length penetrated by radiation is calculated. With this similar information we evaluate the quality of the thermographic profilometry. As thermographic method, optical excited lock-in thermography is used, with halogen lamps for heating, and a cooled MCT camera. With the principle of radiation attenuation in X-ray radioscopy the penetrated length can be calculated for each pixel to be compared to thermographic profilometry. Additional to the depth information, the lateral sizes in radioscopy are given. Also there is a clear geometry without distortions like observed by an optical system. So after performing an image registration between radioscopy and thermography, it is possible to measure lateral sizes in thermography as well, with the additional effect, that many defects and structural information from radioscopy are visualised together with the features detected in thermography. This way one gets an image with more information than one single testing method could provide on its own.

\section{Testing methods}

Two physically different testing methods are used here. One is the optically excited lock-in thermography, which uses defective heat flow for defect sensing. The second method is digital radioscopy, which uses the material absorption of $\mathrm{X}$-radiation and therewith the attenuation of radiation.

\subsection{Optically excited lock-in thermography}

Active thermography involves excitation of heat radiation in objects and its examination with imaging techniques. One makes use of the fact that heat can propagate as a wave. Nevertheless, this wave is exponentially dampened and its propagation speed is strictly frequency dependent [5].

Lock-in thermography applies a periodic excitation, so that the signal can be analysed by Fourier methods to measure amplitude and phase of a thermal wave. The phase signal contains information on the time shift from the excitation to the response. Nevertheless, the phase information of one measurement is not unique. Thus, one needs at least two measurements to determine the depth of a thermal interface. The depth profiling method is worked out in detail later on. 


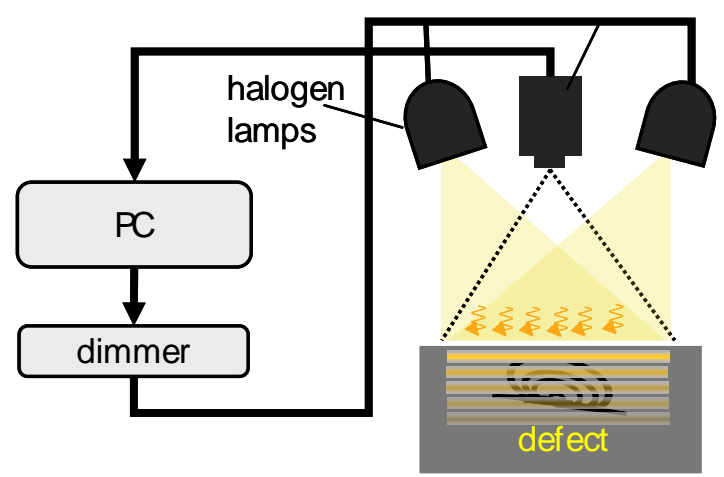

Fig. 1: Schematic diagram of optically exited lock-in thermography

Lock-in thermography is able to resolve surface defects and closed cracks which are difficult to discover with X-ray inspection. It is applicable to delaminations and layer disintegration, to fibre and epoxy nests (in CFRP) as well as to any type of internal layer structure and coating problems with distinguishable thermal effusivities.

Inspection depth is limited by exponential decay of the thermal wave and lateral diffusion. The thermal penetration depth is limited as seen in Eq. 1 with $\alpha \square$ being the temperature diffusivity and $\omega \square$ being the angular frequency (2 $2 \pi$ times the frequency) of the lock-in signal.

$$
\mu=\sqrt{\frac{2 \cdot \alpha}{\omega}}
$$

Typically one reaches a depth of about $3.6 \mathrm{~mm}$ within an inspection time of $100 \mathrm{~s}$ with carbon reinforced plastics. In thermally isotropic materials, the minimal lateral size of visible structures is comparable to their depth. Typically, only one thermal interface is distinguishable, esp. body-to-air interfaces are very prominent and thermally nearly perfectly opaque.

\subsection{Digital projective radioscopy}

Digital radioscopy is a technique to acquire projection images of the specimen using X-rays. With modern radioscopic inspection systems the $X$-rays penetrating the specimen are detected by a digital flat panel detector. Due to the absorption of the tested material, an image of the attenuated X-ray distribution is acquired. In monochromatic cases the attenuated intensity $I$ at a certain point on the image sensor behind the specimen is described in Eq. 2.

$$
I=I_{0} \cdot \mathrm{e}^{-\mu \cdot w}
$$

with $\mu$ as attenuation coefficient, $I_{0}$ as unattenuated $X$-ray intensity and $w$ the penetrated wall thickness. For hidden voids in the specimen the attenuation is weaker, than of the intact specimen (fig. 2 (right)). So the information obtained is from the inside of the specimen but without information on the depth.
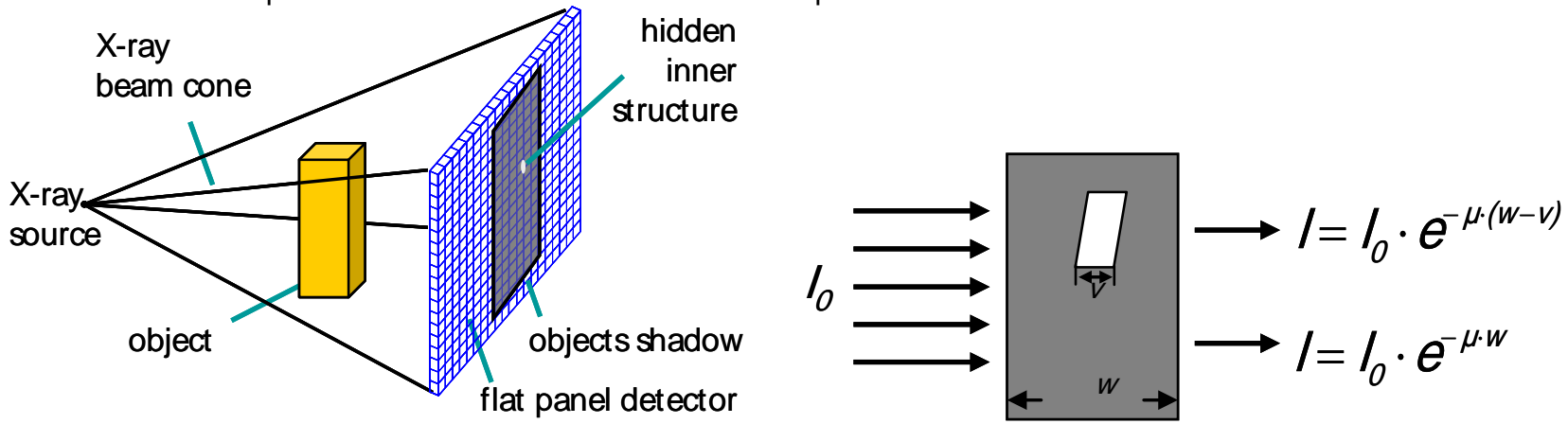

Fig.2: Schematic diagram of digital radioscopy (left), attenuation of $X$-rays in a testing specimen (right)

Since when applying two-dimensional projection imaging all internal structures and details are superimposed in the X-ray image, the contrast of a given defect is getting worse with increasing length of material that has to be penetrated by the 
X-ray beam. E.g. the fully automatic system for inline X-ray inspection ISAR developed at the Fraunhofer Development Center X-ray Technology EZRT is able to detect voids in aluminum castings with a minimum size of approximately $1 \%$ of the total material thickness [1].

This method is well applicable for the investigation of thin CFRP panels. With increasing thickness the absorption rises and the contrast is reduced. In more complex specimen it is impossible to evaluate the different parts of the sample and to distinguish front parts from back parts. So this method is insufficient for complex geometries.

\section{Thermographic depth profiling}

The profilometry done here is based on the model of Busse and Wu for heat dissipation and is extended by a calibration. Therefore calibration measurements at certain lock-in frequencies with a calibration specimen are necessary. The calculated calibration curve applied to the measured data helps to reduce systematic errors.

The model function is dependant on material parameters like the heat capacity $c_{p}$, the density $\rho$ and the thermal conductivity $\lambda$, on the target values like signal amplitude $A$, thermal reflexivity $R$ and depth $d$ and on the modulation frequency of the lock-in technique. These are put in an iterative process with quadratic optimization. (fig. 3). The phase for the model function is aquired from the temperature wave field $\Theta$ in $\mathrm{Eg} .3$

$$
\Theta=A \cdot\left(1+R \cdot e^{\frac{-2 \cdot(1+i) \cdot d}{\mu}}\right)
$$

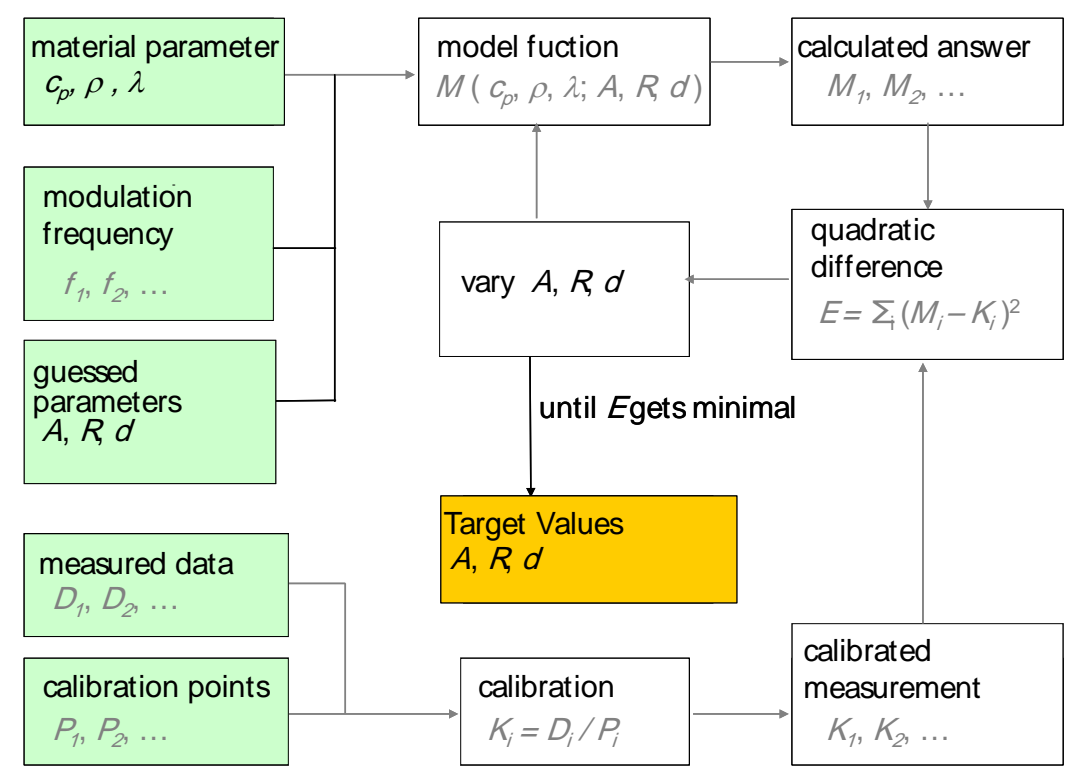

Fig.3: Flowchart of the thermographic depth profiling

\section{Image Registration for multimodal visualisation}

The multimodal visualisation is performed by an image registration based on control points. These corresponding landmarks in both images are selected by hand. The thermography images (phase-, amplitude- and calculated depth image) are all in the same geometry, thus it is only necessary to select the landmarks in the image with the highest contrast. In most cases this is the amplitude image of the optically excited lock-in thermography. Furthermore often a contrast adjustment has to be done in the X-ray and thermography images to find and select control points easily.

With these selected points the images can be registered. As the thermographic images contain mainly surface and surface related information, one of them is registered to the X-ray image, which cannot be transformed without a loss of information. The calculated transformation can be used for the remaining thermographic images later on. Therefore a 
suitable transformation method must be chosen. In the selected examples a projective transform has been applied, to correct the tilt of the thermographic images and to improve the aberrations of the camera's objective.

\section{Results}

In this example a testing panel manufactured of CFRP with drill holes at the reverse side is used. In the registered visualisation one can easily see, that the thermographically calculated depth can be obtained, while by radioscopy the geometric shape is conserved (fig. 4 (left)). In the line profiles (fig. 5) of the wall thickness, calculated radioscopy (fig.4 (middle) and depth profiled thermography (fig.4 (right)), the good shape conservation of radioscopy is obvious, whereas in thermography the defects are more spread the deeper they are.
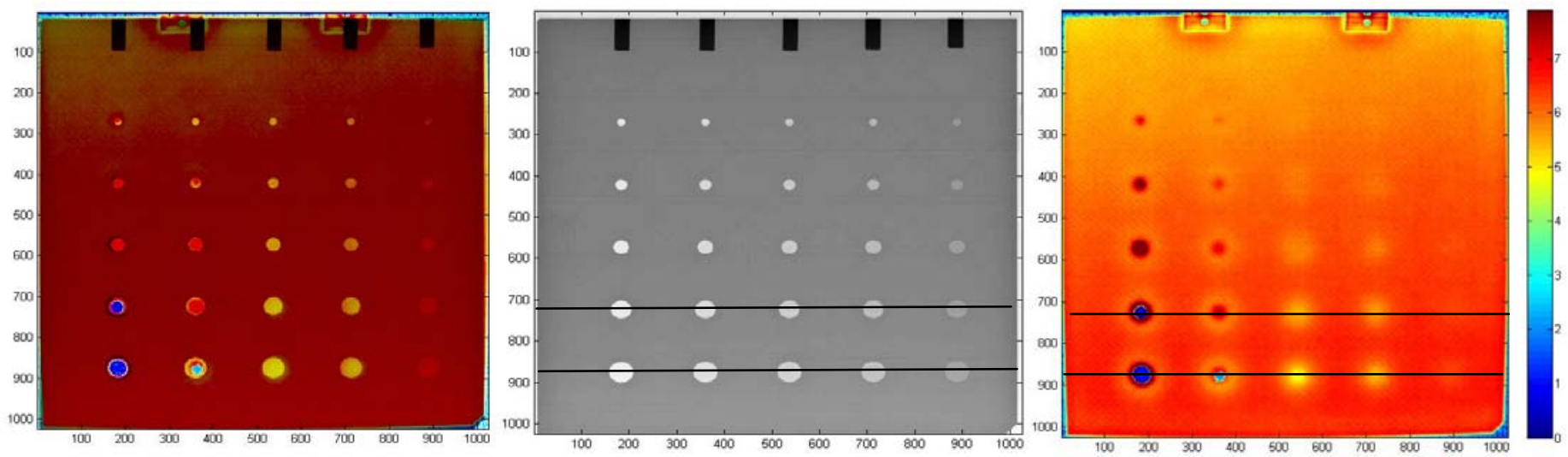

Fig. 4: Registered visualisation of X-ray and thermography (left), X-ray with profile lines (middle), thermographic depth profiled image with profile lines (right)
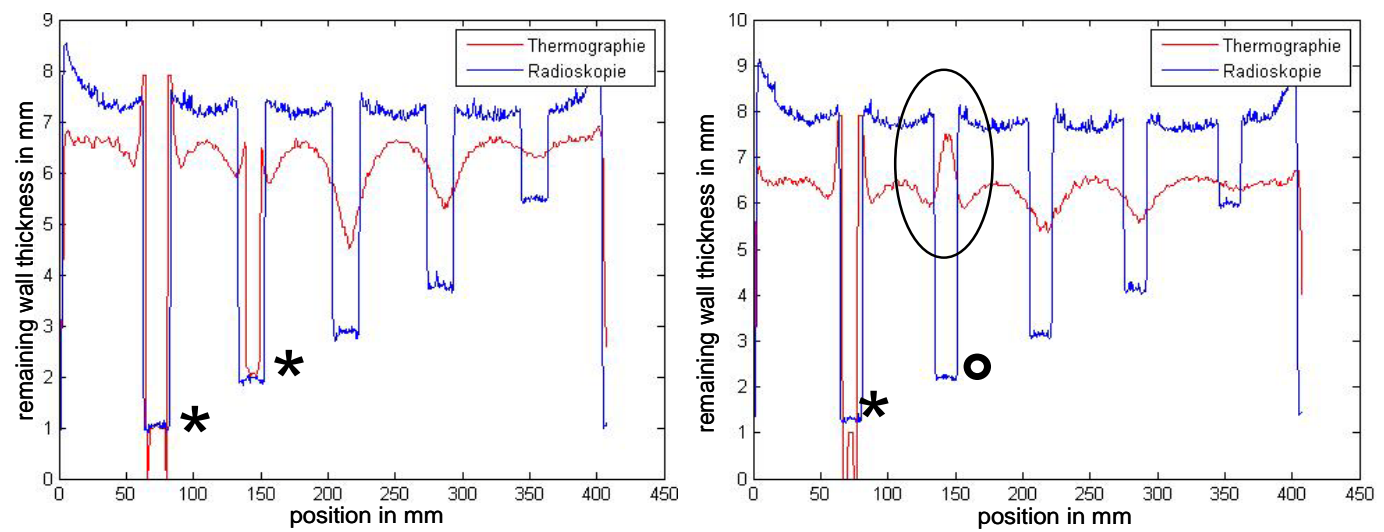

Fig. 5: Profile lines of the lower drill holes (left) and the second row of drill holes (right)

* indicates an excellent match and a good accordance

O indicates a negative calculated depth due to an inverse phase effect

The calculated negative depth in thermography is caused by an inverse phase effect, that is still under investigation, there are still efforts to improve the method. Nevertheless there is a fairly good correlation between the results of radioscopy and thermography. The radioscopy determines the length of the material in beam direction, whereas the thermography provides the depth under the surface of a defect for in this case these values are the same and therefore comparable.

So both methods perfectly complement one other for surface close defects as radioscopy provides the quantity of material, especially how much material is missing and thermography determines the geometric position. Therefore the combination leads to a better defect quantification and provides outstanding advantages in defect analysis. 


\section{Summary and Outlook}

The single testing methods presented herein have advantages and limitations. But by combining the results of each method we can increase the reliability and interpretability in non-destructive testing and therewith the safety. The shown matching allows the calculation of defect geometries and sizes. With the shown techniques the spread of the thermographically observed defects at the surface can be quantified, and a good comparison is possible.

Up to now only two-dimensional methods have been combined, namely radioscopy with quantitative thermography. In the future there will be efforts towards three-dimensional combinations of X-ray computed tomography, as well as an automatic 3-D defect analysis.

\section{REFERENCES}

[1] A. Gleiter, C. Spiessberger, G. Busse: "Phase Angle Thermography for depth resolved defect characterization" in: QIRT 2008 proceedings, S. 435

[2] C. Spiessberger, A. Gleiter, G. Busse: "Data Fusion of Lockin-Thermography Phase Images" in: QIRT 2008 proceedings, S. 483

[3] C. Zöcke, A. Langmeier, R. Stößel, W. Arnold: "A new technique to reconstruction of the defect shape from Lock-in theromography phase images" in: QIRT 2008 proceedings, S. 507

[4] T.M. Buzug, Computed Tomography: From Photon Statistics to Modern Cone-Beam CT, Springer, 2008

[5] G. Busse, D. Wu, W. Karpen, "Thermal wave imaging with phase sensitive modulated thermography." in L.Appl.Phys. Vol. 71, (1992) pp.3962-3965 